\title{
Eyes See what the Mind Knows: Clues to Pattern Recognition in Single Enzyme Deficiency-Related Peroxisomal Disorders
}

\author{
Veronica Arora Sunita Bijarnia-Mahay Sudhisha Dubey Renu Saxena \\ Institute of Medical Genetics and Genomics, Sir Ganga Ram Hospital, New Delhi, India
}

\section{Established Facts}

- Disorders of peroxisomes include inherited defects of peroxisomal biogenesis as well as few single enzyme defects.

- Acyl CoA oxidase (ACOX1) and D-bifunctional protein (DBP) deficiencies are 2 single enzyme defects in the beta-oxidation pathway of very long-chain fatty acids. Clinical profile of the patients is similar to peroxisomal biogenesis disorders, with some biochemical and radiological differences.

\section{Novel Insights}

- ACOX1 and DBP deficiencies not only show similarity in the clinical presentation, but also in facial dysmorphology and neuroradiological features.

- A clinically recognizable facial phenotype and a strikingly similar radiological profile (MRI pattern) can help identify cases of ACOX1 and DBP deficiencies earlier.

- Expansion of the genotypic spectrum with a description of 3 homozygous novel mutations (HSD17B4: c.670C>T, c.1807T>C; ACOX1: 1.03-kb exonic deletion).

\section{Keywords}

Acyl CoA oxidase $\cdot$ Cerebellar ataxia $\cdot$ Cerebellar white matter · D-bifunctional protein · Facial dysmorphism · India · Peroxisomal disorders $\cdot$ Regression

\begin{abstract}
Peroxisomal disorders are a heterogeneous group of inborn errors of metabolism that result in impaired function of the peroxisome. Within this, single enzyme deficiencies are known to cause a constellation of symptoms not very different from the peroxisome biogenesis defects. Thus, there is a need to identify features that differentiate the two. We pres-
\end{abstract}

ent 3 molecularly confirmed families: 1 with Acyl CoA oxidase deficiency and 2 with D-bifunctional protein deficiency. The clinical, biochemical, and radiological features of these patients have been discussed. We attempt to highlight the overlap in facial features as well as strikingly similar MRI findings of cerebellar atrophy and white matter hyperintensities. This unique clinical profile will not only help in reaching a quick diagnosis, but in this era of variants of uncertain significance, it will prove as supporting evidence. Finally, we expand the genotypic spectrum with a description of 3 homozygous novel mutations (HSD17B4: C.670C > T, c.1807T>C; ACOX1: $1.03-\mathrm{kb}$ exonic deletion) and discuss the role of protein modeling its establishing pathogenicity.

(c) 2020 S. Karger AG, Base

Sunita Bijarnia-Mahay

Institute of Medical Genetics and Genomics

Sir Ganga Ram Hospital, Rajinder Nagar

New Delhi 110060 (India)

bijarnia@gmail.com 


\section{Introduction}

Peroxisomal disorders are a heterogeneous group of inborn errors of metabolism that result in impaired function of the peroxisome. They are further subdivided into peroxisomal biogenesis disorders, where there is a "panaffection" of the peroxisomal function or functional deficiencies due to any single enzyme/transporter defect [Aubourg and Wanders, 2013; Wanders et al., 2017]. Acyl CoA oxidase (ACOX1) is the first enzyme in the pathway of beta-oxidation of fatty acids in the peroxisomes. Its deficiency, also known as pseudo-neonatal adrenoleukodystrophy, is caused by biallelic pathogenic variants in the ACOX1 gene. The clinical manifestations are hypotonia, global developmental delay or regression, facial dysmorphism, and occasional organomegaly [Ferdinandusse et al., 2007]. D-bifunctional protein (DBP) catalyzes the second and third step of peroxisomal beta-oxidation of fatty acids and their derivatives. Pathogenic variants in the HSD17B4 gene are known to cause a severe autosomal recessive disorder of $\mathrm{D}$-bifunctional enzyme deficiency [Möller et al., 2001]. The deficiency leads to the accumulation of very long-chain fatty acids (VLCFA) and consequent cell injury. Patients present a severe infantile-onset disorder. The majority succumbs before 2-3 years of life. The clinical course is similar to Zellweger syndrome and is characterized by hypotonia, poor sucking, craniofacial anomalies, hepatomegaly, and intractable seizures. In contrast to Zellweger syndrome, the dysmorphic features are rather subtle or indistinct [Wanders and Waterham, 2006]. Though these disorders have nonspecific neurological phenotypes which can be attributed to numerous other conditions, certain clinical and radiological clues are helpful for recognition.

We discuss the clinical, biochemical, radiological and genetic aspects of these 2 disorders in 3 unrelated families with a molecularly confirmed diagnosis. We provide a simple, yet useful combination of facial gestalt and radiological features which are characteristics of these disorders that helped us to diagnose the second family due to striking similarities with the first.

\section{Case Reports}

Family 1

A 5-year-old boy, third born to nonconsanguineous parents, presented with global developmental delay, regression of previously attained milestones, and a progressive increase in muscle tone since the age of 4 years. After an uneventful antenatal and perinatal period, a delay was noted in all milestones. He sat with support at 9 months of age, started standing at 18 months, walking at 2 years, spoke 2-3 meaningful words at 2 years, and recognized his parents at 9 months. After 26 months of age, a gradual loss of the achieved milestones was noted. There were no triggering signs, such as an acute infection or trauma. Motor milestones were lost earlier followed by language and cognition. He developed progressive stiffening of the lower limbs followed by the upper limbs. There was no history of vision or hearing abnormalities and no history of irritability or inconsolable crying, rapidly increasing head size, or excessive startle.

On examination, facial dysmorphism was noted including frontal bossing, hypertelorism, broad forehead, low nasal bridge, low-set ears, cupid's bow, thick lips and microretrognathia, higharched palate, cryptorchidism, and micropenis (Fig. 1). Anthropometry was within normal limits. Neurological examination revealed gaze-evoked nystagmus, spasticity (more pronounced in the lower limbs), brisk deep tendon reflexes, and a bilateral extensor plantar response. Otherwise, the systemic examination was unremarkable. Among investigations, the complete blood count, plasma lactate, ammonia, dried blood spot assay using tandem mass spectrometry for acyl-carnitines and amino acids were normal. Fundus examination was not contributory. Electroencephalograph revealed generalized epileptiform discharges. Serum transferrin isoforms by isoelectric focusing revealed a normal pattern. MRI findings were suggestive of periventricular as well as cerebellar white matter hyperintensities, cerebellar atrophy as well as cystic areas in the cerebellum (Fig. 2, top row).

Genetic testing by whole-exome sequencing (WES) revealed a novel homozygous variant in the HSD17B4 gene, c.670C>T; (p.Arg224Trp). The variant was noted to be conserved across species and predicted to be damaging by in silico prediction software. It was Sanger validated and was found in a heterozygous state in the parents (Fig. 3B). As this was a variant of uncertain significance, further supporting evidence was required. Analysis of VLCFA was performed which showed elevated levels of pristanic acid: $0.52(<0.28)$ as well as a high ratio of C24/C22 $1.07(0.74-1.02)$, ratio C26/C22 0.09 (0.006-0.02).

Protein prediction software [Venselaar et al., 2010], Project HOPE, was used to study the effect of the variant (Fig. 3A). It was inferred that the mutant residue is bigger than the wild-type residue, and the wild-type residue charge was positive, the mutant residue charge is neutral. Also, the mutant residue is more hydrophobic than the wild-type residue. These differences are likely to disrupt the protein structure and were supporting criteria for our analysis. The pathogenicity of the variant was thus established consistent with the ACMG criteria [Richards et al., 2015], achieving a molecular confirmation of D-bifunctional enzyme deficiency in the child. The parents were counseled, and the child's supportive treatment was continued.

\section{Family 2}

A 4-year-old girl, first born of a consanguineous marriage, presented with global developmental delay, noted since her infancy, and slow progressive loss of acquired skills noticed since 3.5 years of age. She was born at term after an uneventful antenatal and perinatal period. She sat with support at 10 months, started standing at 16 months, walking at 2 years of age, and spoke 2-3 meaningful words at 2 years. She had started recognizing her parents at 9 months of age. They noted a regression of milestones after approximately 3 years 4 months of age. The motor milestones were 
Fig. 1. A, B Front views of patient 1 (A) and patient 2 (B) showing frontal bossing, hypertelorism, broad forehead, low nasal bridge, low-set ears, cupid's bow, thick vermilion border of lower lips, and micrognathia.
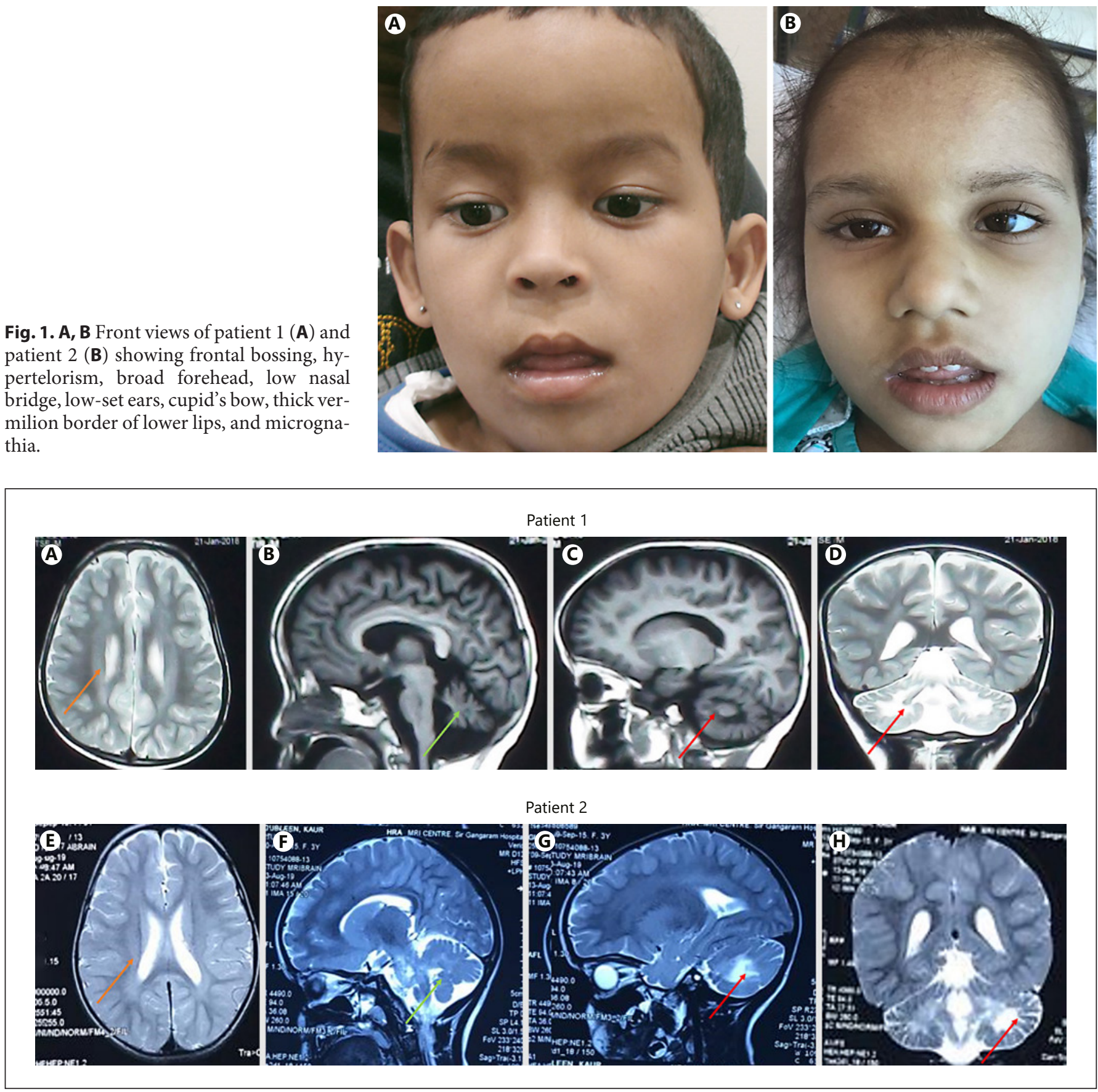

Fig. 2. MRI images of patient 1 (top) and patient 2 (bottom). Note the striking similarity between the findings among the 2 patients. A, E Axial T2-weighted images showing bilateral periventricular white matter changes (orange arrows). B, F Sagittal images show-

lost earlier, followed by language and cognition. They also noticed a decreased tone in all the limbs. There was no history of vision or hearing abnormalities. There was a history of a similarly affected younger sibling who died at the age of 2.5 years.

ACOX1 and D-Bifunctional Protein

Peroxisomal Disorders: Pattern

Recognition

ing cerebellar atrophy (green arrows). C, G Sagital images showing cerebellar white matter changes (red arrows). D, H Coronal images showing bilateral cerebellar symmetrical white matter hyperintensities (red arrows).

On examination, she was interactive with frequent smiles to her mother. Facial dysmorphism included a long face, frontal bossing, hypertelorism, broad forehead, low nasal bridge, low-set ears, cupid's bow, thick lips, and microretrognathia (Fig. 1B)

Mol Syndromol 2020;11:309-314 


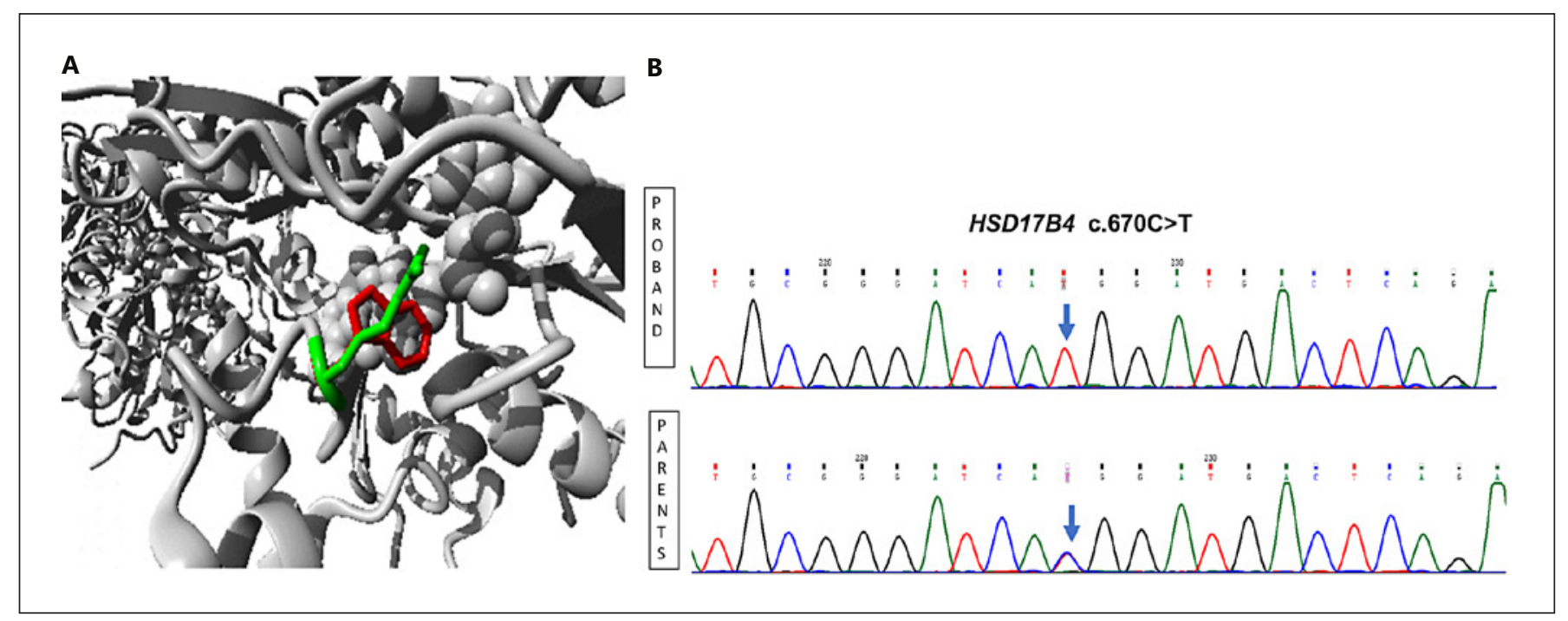

Fig. 3. A The protein structure of the D-bifunctional protein with the wild-type residue in green and mutant in red for the variant in patient 1. B Chromatographic representation of Sanger sequencing showing the variant in patient 1 (HSDB17B4: c.670C>T). The upper sequence shows the variant in the homozygous form (blue arrow), and the lower sequence shows the variant present in a heterozygous form (blue arrow).

Brain MRI showed cerebellar atrophy, cerebellar white matter changes with cystic lesions (Fig. 2F, G). As the clinical course, facial gestalt and MRI findings were very similar to the previous patient with DBP deficiency, a VLCFA was requested. It revealed high phytanic acid, $6.99(0.38-5.04 \mathrm{mmol} / \mathrm{L})$, and high pristanic acid, $0.79(0.11-0.43 \mu \mathrm{mol} / \mathrm{L})$, while docosanoic, C22:0-47.74 (36.9$82.6 \mu \mathrm{mol} / \mathrm{L})$ and tetracosanoic acid, C24:0-50.08 (32.1-71.9 $\mu \mathrm{mol} / \mathrm{L})$ were normal. Hexacosanoic was high C26:0-3.96 (0.35$1.22 \mu \mathrm{mol} / \mathrm{L})$, with a high ratio of $\mathrm{C} 24 / \mathrm{C} 221.05(0.74-1.02)$ and C26/C22 $0.08(0.006-0.02)$. The ratio of pristanic/phytanic acids was normal, $0.11(0.071-0.311)$. Targeted genetic testing revealed a novel 1.03-kb homozygous deletion in exon 3 of the ACOX1 gene (chr17:g.73955865-73956891del). The same was present in a heterozygous state in the parents and is a pathogenic variant according to the ACMG classification [Richards et al., 2015]. DNA of the other affected sibling was not available for testing.

Family 3

Two male siblings, aged 2 and 5 years, born to nonconsanguineous parents presented with recurrent seizures and global developmental delay. Both boys had very similar problems of early-onset intractable seizures (day 2 of life) with very severe global developmental delay and hypotonia. Both were bedridden, were not able to recognize their parents, and had failure to thrive. There was no major dysmorphism and no feeding problems. The second child was noted to have liver damage in the preterminal stage. EEG was normal. CT scan revealed white matter changes in the cerebellum and cerebral parenchyma as well as cerebellar atrophy. The couple presented to the genetic clinic after the death of the children for preconception counseling. In absence or nonavailability of DNA from any of the affected children with a seemingly autosomal recessive genetic disorder, WES was performed on the couple.
WES revealed the couple to be carriers of a novel variant, c.1807T>C; (p.Trp603Arg) in the HSD17B4 gene. The variant is likely pathogenic according to the ACMG classification (PM1, PM2, PP3, PP4).

\section{Discussion/Conclusion}

DBP and ACOX1 enzymes carry out 2 successive steps of peroxisomal beta-oxidation. The ACOX1 enzyme carries out the first step of beta-oxidation of saturated VLCFA, polyunsaturated fatty acids, and dicarboxylic acids, but not branched-chain fatty acids or bile acid intermediates. The primary role of DBP is beta-oxidation of VLCFAs, pristanic acid, and di- and trihydroxycholestanoic acids. Their deficiencies cause 2 rare autosomal recessive and similar disorders and should be suspected in all infants with neuroregression and typical neuroimaging findings [Watkins et al., 1995]. In these disorders, the changes related to demyelination are seen in the cerebellum and also involve the brainstem, posterior limb of the internal capsule, and posterior cerebral white matter [Suzuki et al., 2002; Ferdinandusse et al., 2006; Grønborg et al., 2010; Poll-The and Gärtner, 2012]. We describe remarkable similarities in neuroimaging findings of confluent cerebellar cystic changes in 3 patients with peroxisomal defects due to 2 types of single enzyme deficiencies 
(Fig. 2). These striking changes can be an important diagnostic clue for this disorder and have been previously described as a "peroxisomal pattern" [Grønborg et al., 2010]. Other features in the peroxisomal pattern apart from cerebral and cerebellar leukoencephalopathy changes are the perisylvian polymicrogyria and pachygyria, which were not found in our cases.

The clinical profile of the 2 children (patients 1,2) described nonspecific global developmental delay and regression. Strikingly, similar facial gestalt (Fig. 1) in these children is an important feature which can be used to narrow down the list of differentials. Common features including a prominent forehead, frontal bossing, scanty scalp hair, hypertelorism, sparse eyebrows, prominent nasal bridge, low-set ears, a long prominent philtrum, tented upper lip, downturned corners of the mouth, and full lower lips were identified in both children. In the study by Ferdinandusse et al., [2006], facial dysmorphism was noted in $68 \%$ of the patients [Ferdinandusse et al, 2006]. This pattern of dysmorphism is distinctive and reminiscent of a peroxisomal biogenesis disorder. However, it has not been described before comparing the 2 enzyme deficiency disorders. Facial recognition software such as Face2gene could be used to train this skill and help manage clinical diagnosis [Bijarnia-Mahay and Arora, 2019]. It is interesting to note that in spite of differences of the substrate specificity between ACOX1 and DBP (namely the branched-chain fatty acids including the side chain of the bile acid intermediates DHCA/THCA), the MRI and the clinical similarities are so prominent.

MRI abnormalities in peroxisomal disorders are variable and range from neuronal migration defects to delayed myelination of the cerebellum, cerebral hemispheres, cerebral atrophy, and hypoplastic corpus callosum. Ferdinandusse et al. [2006] described 125 patients of D-bifunctional enzyme deficiency, and the most common MRI features included generalized cortical atrophy, cortical dysplasias, and cerebellar hypoplasia. Cerebellar germinolytic cysts resembling those found in our patients were the most specific findings. Early MRI findings of ACOX1 deficiency also include cerebral and cerebellar white matter changes [Suzuki et al., 2002; Kurian et al., 2004]. Another patient with identical MRI findings of extensive signal abnormalities in brain stem tracts, middle cerebellar peduncles, and the cerebellar white matter has been reported, similar to our patient [Suzuki et al., 2002; Poll-The and Gärtner, 2012].

Thus, this pattern of neuroimaging findings is quite specific for ACOX1/DBP deficiency and warrants a biochemical confirmation using VLCFA analysis. Further, we strongly advocate VLCFA analysis for children with tone abnormalities and cerebellar cystic anomalies. In the era of next-generation sequencing, such tests not only save time and money, but also serve as a functional basis for these disorders. This is especially true in cases of novel variants, which are often labeled as variants of uncertain significance, making a definitive diagnosis and prenatal diagnosis a challenge. It is important to recognize such rare disorders in light of emerging gene therapies an example being that of X-linked adrenoleukodystrophy [Eichler et al., 2017].

In conclusion, we report 3 families with novel pathogenic variants in genes encoding for single peroxisomal enzymes. We aim to highlight a unique constellation of clinical and neuro-radiological features which would help to confim the diagnosis and guide testing in the right direction.

\section{Acknowledgement}

The authors would like to acknowledge and thank the patients' families for participating in this study.

\section{Statement of Ethics}

The patients' parents gave their written informed consent to publish their case (including publication of images). Ethics approval was not taken in for this case report. The paper is exempt from ethical committee approval since all the patients were seen and tested as per standard of care, and the study was undertaken as a retrospective descriptive study.

\section{Conflict of Interest Statement}

The authors have no conflicts of interest to declare.

\section{Funding Sources}

This study and manuscript was unfunded.

\section{Author Contributions}

V.A. wrote the manuscript and assisted in clinical management. S.B.M. managed the case and supervised in writing the manuscript. R.S. and S.D. performed the molecular analysis after nextgeneration sequencing in all the 3 families. 


\section{References}

Aubourg P, Wanders R. Peroxisomal disorders. Handb Clin Neurol. 2013;113:1593-609.

Bijarnia-Mahay S, Arora V. Next generation clinical practice - It's man versus artificial intelligence! Indian Pediatr. 2019;56(12):1007-8.

Eichler F, Duncan C, Musolino PL, Orchard PJ, De Oliveira S, Thrasher AJ, et al. Hematopoietic stem-cell gene therapy for cerebral adrenoleukodystrophy. N Engl J Med. 2017; 377(17):1630-8.

Ferdinandusse S, Denis S, Mooyer PA, Dekker C, Duran M, Soorani-Lunsing RJ, et al. Clinical and biochemical spectrum of D-bifunctional protein deficiency. Ann Neurol. 2006;59(1): 92-104.

Ferdinandusse S, Denis S, Hogenhout EM, Koster J, van Roermund CW, IJlst L, et al. Clinical, biochemical, and mutational spectrum of peroxisomal acyl-coenzyme A oxidase deficiency. Hum Mutat. 2007;28(9):904-12.

Grønborg S, Krätzner R, Spiegler J, Ferdinandusse S, Wanders RJ, Waterham HR, et al. Typical cMRI pattern as diagnostic clue for D-bifunctional protein deficiency without apparent biochemical abnormalities in plasma.Am J Med Genet A. 2010;152A(11): 2845-9.
Kurian MA, Ryan S, Besley GT, Wanders RJ, King MD. Straight-chain acyl-CoA oxidase deficiency presenting with dysmorphia, neurodevelopmental autistic-type regression and a selective pattern of leukodystrophy. J Inherit Metab Dis. 2004;27(1):105-8.

Möller G, van Grunsven EG, Wanders RJ, Adamski J. Molecular basis of D-bifunctional protein deficiency. Mol Cell Endocrinol. 2001; 171(1-2):61-70.

Poll-The BT, Gärtner J. Clinical diagnosis, biochemical findings and MRI spectrum of peroxisomal disorders. Biochim Biophys Acta. 2012;1822(9):1421-9.

Richards S, Aziz N, Bale S, Bick D, Das S, GastierFoster J, et al. Standards and guidelines for the interpretation of sequence variants: a joint consensus recommendation of the American College of Medical Genetics and Genomics and the Association for Molecular Pathology. Genet Med. 2015;17(5):405-24.
Suzuki Y, Iai M, Kamei A, Tanabe Y, Chida S, Yamaguchi $\mathrm{S}$, et al. Peroxisomal acyl CoA oxidase deficiency. J Pediatr. 2002;140(1):12830.

Venselaar H, Te Beek TA, Kuipers RK, Hekkelman ML, Vriend G. Protein structure analysis of mutations causing inheritable diseases. An e-Science approach with life scientist friendly interfaces. BMC Bioinformatics. 2010;11:548.

Wanders RJ, Waterham HR. Peroxisomal disorders: The single peroxisomal enzyme deficiencies. Biochim Biophys Acta. 2006; 1763(12):1707-20.

Wanders RJ, Klouwer FC, Ferdinandusse S, Waterham HR, Poll-Thé BT. Clinical and Laboratory Diagnosis of Peroxisomal Disorders. Methods Mol Biol. 2017;1595:329-42.

Watkins PA, McGuinness MC, Raymond GV, Hicks BA, Sisk JM, Moser AB, et al. Distinction between peroxisomal bifunctional enzyme and acyl-CoA oxidase deficiencies. Ann Neurol. 1995;38(3):472-7. 